\title{
Unique presentation of palatal metastasis from high- grade spindle cell sarcoma of the bone
}

\author{
Valentina Fenech, Anton Queen, Archana Gadve, Fiona Cowie
}

Department of Oncology, Beatson West of Scotland Cancer Centre, Glasgow, UK

\section{Correspondence to} DrValentina Fenech; vally.fenech@gmail.com

Accepted 13 October 2020

\section{SUMMARY}

Spindle cell sarcoma (SCS) is a rare malignant tumour which can arise in bone and accounts for $2 \%-5 \%$ of primary bone cancer cases. Distant metastasis occurs predominantly in the lungs. However, metastasis to the soft palate, to the best of our knowledge, has never been previously reported. In this case report, we describe a unique presentation of soft palate metastasis in a patient with a history of high-grade SCS of the bone who presented with progressive dysphagia and nausea and vomiting who underwent surgical excision for palliation of symptoms.

\section{BACKGROUND}

Spindle cell sarcoma (SCS) is a rare malignant tumour which can arise in bone and accounts for $2 \%-5 \%$ of primary bone cancer cases. ${ }^{1}$ We present this unique case of soft palate metastasis from high-grade SCS of bone and an approach to palliation of distressing symptoms in a patient who was otherwise not suitable for active treatments. In our patient, surgical excision of soft palate metastasis was used for management of dysphagia and intractable nausea and vomiting.

\section{CASE PRESENTATION}

A 66-year-old man was diagnosed with high-grade SCS of the proximal right humerus in May 2016. In July 2016, he underwent surgical resection and total humeral endoprosthetic replacement followed by six cycles of adjuvant cisplatin and doxorubicin. He completed treatment in January 2017, and this was followed by a 2-year disease-free interval.

In March 2019, at the age of 68, he developed bilateral pulmonary metastases, which were detected on a surveillance chest X-ray. Initially, he was asymptomatic from this, but a few months later, in September 2019, he developed haemoptysis, which was treated with palliative radiotherapy to the mediastinum with $2000 \mathrm{cGy}$ in five fractions.

In October 2019, he presented with a 3-week history of progressive dysphagia and intractable nausea and vomiting. During this time, he had noted a pedunculated lesion at the roof of his mouth. $\mathrm{He}$ described it as a firm, non-tender lesion which was growing, from the size of a pea and increasing to the size of a large date over a few days. He denied any associated symptoms, specifically oral bleeding. There was no history of preceding trauma in the area or medication changes.

He had no significant dental history. His medical history included asthma, osteoarthritis, peptic ulcer disease, hypertension, axillary vein thrombosis associated with peripherally inserted central catheter insertion and previous pulmonary embolism. He was an active smoker (54 pack-years) and consumed a moderate amount of alcohol. There was no significant family history.

Intraoral examination revealed a large pedunculated mass arising from the soft palate just anterior to the uvula (figure 1). The extraoral examination was normal and regional lymph nodes were nonpalpable. He was admitted for further investigation and management.

\section{INVESTIGATIONS}

A CT of the chest, abdomen and pelvis confirmed the presence of a low-attenuation lesion arising from the posterior aspect of the soft palate and extending into the oropharynx. The lesion measured approximately $22 \times 16 \times 20 \mathrm{~mm}$ (anteroposterior $\times$ transverse $\times$ craniocaudal) and appeared separate from the base of the tongue. There was no evidence of deep extension or cervical lymphadenopathy (figure 2). Unfortunately, the CT scan also demonstrated evidence of progressive disease with interval enlargement of known pulmonary metastases and evidence of new pulmonary lesions. A CT of the head and routine blood investigations were unremarkable.

\section{DIFFERENTIAL DIAGNOSIS}

His clinical presentation was highly suspicious of malignancy, and although a primary intraoral tumour or a second separate mesenchymal malignancy in the soft palate was considered as a differential diagnosis, the possibility of a metastatic deposit from previously resected high-grade SCS of the bone could not be ruled out, given the history.

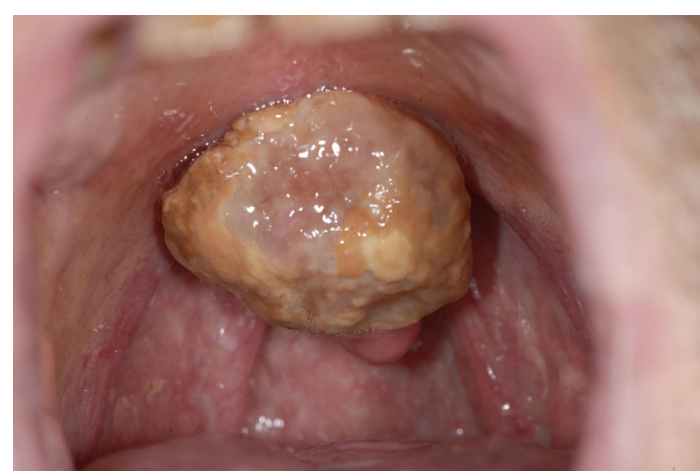

Figure 1 Intraoral examination of soft palate lesion. 


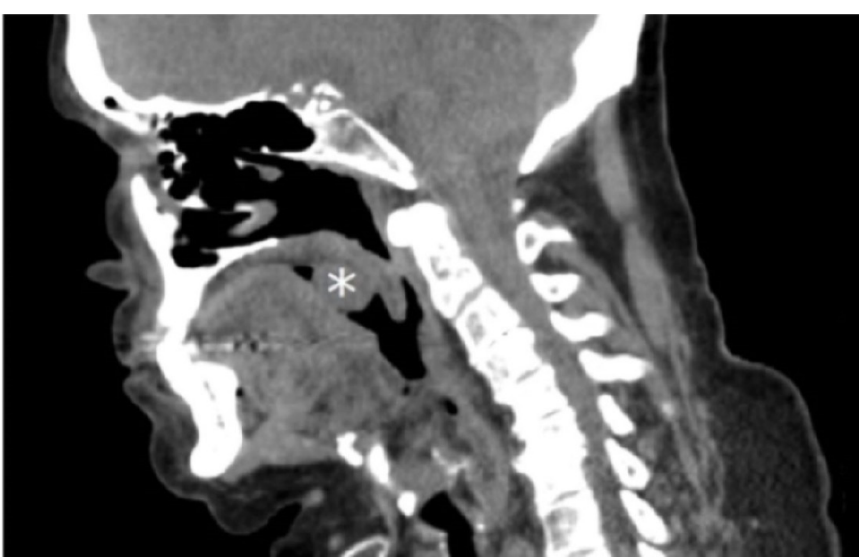

Figure 2 Unenhanced CT image of soft palate lesion $\left(^{*}\right)$ in sagittal view measuring $22 \times 16 \times 20 \mathrm{~mm}$ (transverse $\times a n t e r o p o s t e r i o r \times c r a n i o c a u d a l)$ and separate from the base of the tongue.

\section{TREATMENT}

He was treated with intravenous fluids and regular antiemetics. He was reviewed by the local ear, nose and throat (ENT) team and underwent surgical excision of the soft palate mass under local anaesthesia. There were no intraprocedural or postprocedural complications.

Histopathological examination of the postsurgical specimen showed morphological appearances consistent with a SCS (figure 3). Immunohistochemistry showed positive staining for smooth muscle actin and negative staining for S-100, desmin, AE1/3, MNF, CD34, CD31, CAM5.2 and caldesmon (figure 4). The immunoprofile of this specimen was identical to the previously resected high-grade SCS and therefore in-keeping with relapsed disease.

\section{OUTCOME AND FOLLOW-UP}

Surgery resulted in complete resolution of dysphagia and intractable nausea and vomiting, and the patient was discharged home. Unfortunately, 3 months later in January 2020, he experienced regrowth of the soft palate mass, causing increasing difficulty with swallowing. He was treated with further excision of the soft palate mass by ENT. The patient died from progressive pulmonary metastases 3 years and 9 months from the original diagnosis of SCS.

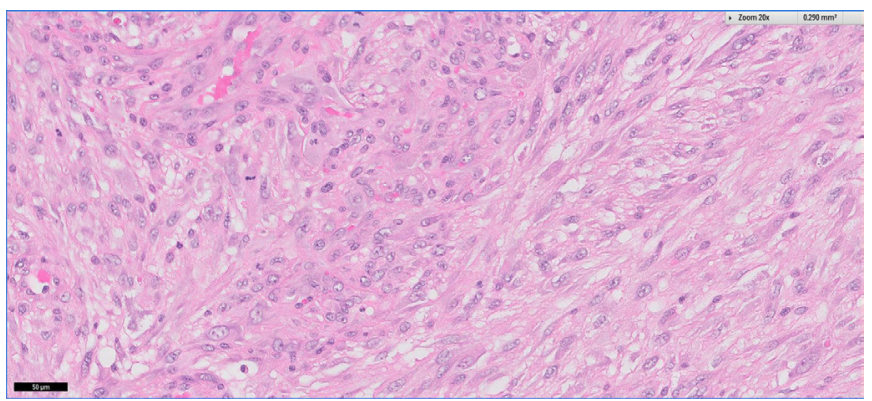

Figure 3 Light microscope image of the soft palate lesion by H\&E staining (magnification $\times 20$ ) shows the tissue is composed of fascicles of spindle-shaped cells with abundant eosinophilic cytoplasm. There is moderate to marked nuclear pleomorphism and prominent mitotic activity, including the presence of abnormal mitosis.

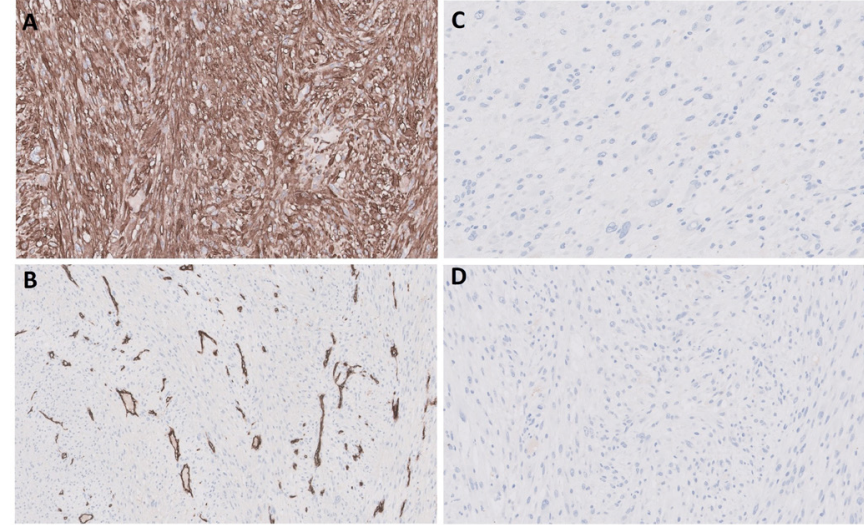

Figure 4 IHC staining patterns of the soft palate lesion for SMA, CD34, AE1/3 and S-100. (A) Positive staining for SMA in the spindled tumour cells. (B) Negative staining of tumour cells for CD34 and evidence of CD34 uptake by normal blood vessel endothelial cells. (C) Negative staining of tumour cells for AE1/3. (D) Negative staining of tumour cells for S-100. The IHC staining patterns for desmin, MNF, CD31, CAM5.2 and caldesmon show identical features to microscopic images C and D. IHC, immunohistochemical; SMA, smooth muscle actin.

\section{DISCUSSION}

SCS is a small heterogeneous group of primary malignant mesenchymal tumours that may occur in the bone. SCS is rare and accounts for approximately $2 \%-5 \%$ of all primary bone cancers. ${ }^{1}$

The main types of SCS of bone include fibrosarcoma (FS), leiomyosarcoma, angiosarcoma and undifferentiated pleomorphic sarcoma, which was previously known as malignant fibrous histiocytoma. $^{2-4}$ In general, SCS of the bone appears to have a male predominance and often affects more older patients than osteosarcoma (OS). ${ }^{4}$ The development of SCS of bone may occur in patients with a previous history of bone disease such as Paget's disease or therapeutic radiation exposure. ${ }^{2-4}$ Many SCSs occur in the long bones of the lower limbs and patients usually

\section{Patient's perspective}

I remember that it started out as a small bump, about 2-3 $\mathrm{mm}$ in size, towards the back of the roof of the mouth. It felt like a pimple against my tongue and looked like a small red spot in the mirror. At this point I felt fine and brushed it off and carried on with my life as normal.

But over the next few days, I noticed that it had started to increase in size. It was almost as if it was growing slightly bigger each day. I could feel that there was something rough and dry growing at the back of my mouth, catching the back of my throat and making me feel uncomfortable. I then began to notice that I was having difficulty swallowing solid foods like steak and pork. It got to the point that every time I ate or drank something it was causing me to gag and vomit. When I looked in the mirror, I was shocked to see something I thought looked like an alien or a second uvula.

I was admitted to hospital where I was told that I had a lump growing from the roof of my mouth. I spent a few days in the hospital undergoing treatment and had the lump taken out by one of the ear, nose and throat surgeons under local anaesthetic. Luckily, the operation was successful and I can go back to eating and drinking normally again. 
Learning points

- Spindle cell sarcoma (SCS) of the bone is a rare type of malignant tumour which predominantly metastasises to the lungs and bone.

- All patients with a suspect diagnosis of SCS should be managed by a specialised sarcoma multidisciplinary team.

- Surgical palliation is an effective intervention in patients with metastatic disease to alleviate distressing symptoms and to improve quality of life.

present with pain, and there is a high incidence of pathological fractures. ${ }^{2}$

The management of SCS of the bone is similar to that for OS and usually involves chemotherapy and complete surgical excision, usually limb-salvage or amputation, under the supervision of a specialised bone sarcoma multidisciplinary team. ${ }^{2}$ SCSs of the bone are usually high-grade tumours, and patients are at a significant risk of relapse during follow-up with local recurrence or distant metastases. ${ }^{4-7}$ Distant metastases occur most commonly to the lungs and bone. ${ }^{4-7}$ However, metastasis to the soft palate, to the best of our knowledge, has never been previously reported.

The management of progressive and unresectable disease can be challenging and is usually guided by the burden of symptoms and potential toxicity of treatment. In some selected cases of metastatic disease, surgical resection can be considered for symptom control. ${ }^{1}$ In this case, early referral to the local ENT team for consideration of surgical resection of the soft palate mass for palliation of symptoms was the most appropriate option. The mass was excised successfully and the patient regained the ability to eat and drink normally.

SCS of the bone is generally associated with a poor overall survival outcome and the presence of a tumour size greater than
$9 \mathrm{~cm}$, age greater than 40 years, axial tumour site, FS subtype and pathological fracture are all negative prognostic factors. ${ }^{4}$

Acknowledgements The authors thank Dr Jeff White, consultant medical oncologist at the Department of Oncology, Beatson West of Scotland Cancer Centre, Glasgow, UK, for his support and general supervision; Dr Elaine MacDuff, consultant pathologist at the Department of Pathology, Queen Elizabeth University Hospital, Glasgow, UK, for providing the histological diagnosis; and Mr Alexandros Tsikoudas, consultant ENT surgeon at the Department of ENT, Gartnavel General Hospital, Glasgow, UK, who carried out the surgical intervention.

Contributors VF and AQ: planning and writing the manuscript and literature review; AG review of the full text and critical revision of the work; FC: final approval and review of the work.

Funding The authors have not declared a specific grant for this research from any funding agency in the public, commercial or not-for-profit sectors.

Competing interests None declared.

Patient consent for publication Obtained.

Provenance and peer review Not commissioned; externally peer reviewed.

\section{REFERENCES}

1 Gerrand C, Athanasou N, Brennan B, et al. Uk guidelines for the management of bone sarcomas. Clin Sarcoma Res 2016;6:7.

2 Hogendoorn PCW, Athanasou N, et al, ESMO/EUROBONET Working Group. Bone sarcomas: ESMO clinical practice guidelines for diagnosis, treatment and follow-up. Ann Oncol 2010;21 Suppl 5:v204-13.

3 Chen K-H, Chou T-M, Shieh S-J. Management of extremity malignant fibrous histiocytoma: a 10-year experience. Formos J Surg 2015;48:1-9.

4 Berner K, Johannesen TB, Hall KS, et al. Clinical epidemiology and treatment outcomes of spindle cell non-osteogenic bone sarcomas - a nationwide population-based study. J Bone Oncol 2019;14:100207.

5 Bielack SS, Schroeders A, Fuchs N, et al. Malignant fibrous histiocytoma of bone: a retrospective EMSOS study of 125 cases. Acta Orthop Scand 1999;70:353-60

6 Brewer P, Sumathi V, Grimer RJ, et al. Primary leiomyosarcoma of bone: analysis of prognosis. Sarcoma 2012;2012:1-4

7 Picci P, Bacci G, Ferrari S, et al. Neoadjuvant chemotherapy in malignant fibrous histiocytoma of bone and in osteosarcoma located in the extremities: analogies and differences between the two tumors. Ann Oncol 1997:8:1107-15.

Copyright 2020 BMJ Publishing Group. All rights reserved. For permission to reuse any of this content visit

https://www.bmj.com/company/products-services/rights-and-licensing/permissions/

BMJ Case Report Fellows may re-use this article for personal use and teaching without any further permission.

Become a Fellow of BMJ Case Reports today and you can:

- Submit as many cases as you like

- Enjoy fast sympathetic peer review and rapid publication of accepted articles

- Access all the published articles

- Re-use any of the published material for personal use and teaching without further permission

Customer Service

If you have any further queries about your subscription, please contact our customer services team on +44 (0) 2071111105 or via email at support@bmj.com.

Visit casereports.bmj.com for more articles like this and to become a Fellow 